\title{
Immune Responses Mediated by Th17 Cells in Helicobacter pylori Infection
}

\author{
Chang Liu $^{a} \quad$ Zhengli Zhang $^{\text {b }}$ Meiping Zhu ${ }^{b}$ \\ ${ }^{a}$ College of Basic Medicine, and bepartment of Gastroenterology, Shuguang Hospital, \\ Shanghai University of Traditional Chinese Medicine, Shanghai, China
}

\author{
Key Words \\ Helicobater pylori $\cdot$ Th17 cell $\cdot$ T helper cells $\cdot$ Mucosal immunity
}

\begin{abstract}
T helper 17 (Th17) cells are one of the CD4+ T-cell subsets which induce a variety of diseases by secreting IL-17 and other inflammatory factors. After Helicobacter pylori ( $\mathrm{Hp}$ ) infection, cytotoxin-associated gene $A$ and urease subunit $B$ regulate the number of Th17 cells via induction of cell differentiation by infected macrophages, activation of MyD88 and other pathways, and by driving chemokines, which upregulates the number of both Th17 cells and regulatory T cells (Tregs) and switches towards a Treg-type immune response. Meanwhile, Th17 cells also play important roles in the response to $\mathrm{Hp}$ infection, participating in the clearance of $\mathrm{Hp}$ by recruiting neutrophils and expanding inflammatory response, causing mucosal damage and even inducing cancer. Thereby, Th17 cells participate in the occurrence and development of Hp-related diseases, such as gastritis, peptic ulcer disease and gastric cancer.
\end{abstract}

(C) 2016 The Author(s)

Published by S. Karger AG, Basel

\section{Introduction}

Helicobacter pylori (Hp), a sort of Gram-negative microaerobic bacterium, is one of the main risk factors for gastritis and peptic ulcer disease and closely related to gastric carcinoma. It is able to evade the host's immune response and induce persistent inflammation. It is already known that Hp inflammation can induce an imbalance of T helper 1 (Th1)/Th2 cells in gastric mucosa [1], and now, the imbalance of Th17 cells/regulatory T cells (Tregs) is also proposed [2]. T-cell immunity plays an important role after inflammation and even affects the prognosis of the disease. Here, we briefly introduce the role which Th17 cell-mediated immune response plays in Hp inflammation. 


\section{Th17 Cells}

T cells play a central role in immunity and are divided into Th cells, Tregs and cytotoxic T cells; among these, Th cells are differentiated by CD4+ T cells, a subset assisting the response of T and B cells. Subsets of Th cells, including Th1, Th2, Th17, Th9, Th22 and Tfh, stay in equilibrium under normal circumstances; however, they differentiate into a variety of Th cells after the stimulation of antigens, cytokines and antigen-presenting cells. Different subsets of Th cells can also differentiate into each other.

As a subset of CD4+ T cells, Th17 cells not only participate in the innate immune response against bacteria and fungi but also secrete cytokines like IL-17, IL-22 and IL-6, thus inducing a variety of autoimmune diseases. IL-17, being considered as one of the most important cytokines, is divided into IL-17A (usually referred to as IL-17), IL-17B, IL-17C, IL-17D, IL-17E (IL-25) and IL17-F, which play important roles in the immune response. It can induce the production of granulocyte-macrophage colony-stimulating factor, peptides, endothelial and epithelial cells, chemokines, cytokines and metalloproteinases (as well as others), the recruitment and activation of neutrophils, and also participate in the inflammatory reaction [3]. It is well known that retinoid-related orphan receptor gamma t (ROR $\gamma \mathrm{t}$ ) is the master transcription factor for the differentiation of Th17 cells; concomitant IL-6 and transforming growth factor (TGF)- $\beta$ occupy an important position in the beginning of differentiation, whereas other cytokines like IL-23 are involved in the maintenance and proliferation phase.

\section{Hp Infection Affects Th17 Cells}

Hp can exist in the host body for a long time due to immunologic escapement. Mucosal CD4 T-cell numbers increase following Hp infection after 4 weeks [4], despite a lack of ongoing HP infection, while HP-specific Th17 cells persist in the blood and gastric mucosa of individuals with a past HP infection [5]. This suggests that Hp infection induces a mucosal inflammatory reaction in the very beginning, and, thus, the reaction runs through the whole pathogenesis.

Several studies have shown the involvement of Hp-associated proteins in Th17 cell response. Cytotoxin-associated gene A (CagA), an immunodominant antigen subsistent in the highly virulent strain of $\mathrm{Hp}$, contributed to the ability of Hp to evade Th17 cell-mediated clearance by modulating the expression of B7-H2 [6]. Meanwhile, urease subunit B (UreB), a protective antigen of $\mathrm{Hp}$, induced Th17 cell response. Cocultured splenic lymphocytes from Hp-infected mice with the recombinant UreB (rUreB) elevated IL-17 secretion and caused an increase in the number of Th17 cells. The expression of IL- 6 and IL-23p19 was significantly increased in rUreB-stimulated macrophages. Whole-cell protein of a UreB-deficient strain induced less Th17 cell responses than that of the wild-type strain. In addition, subcutaneous and intranasal immunization of rUreB elicited antigen-specific Th17 cell responses. Intranasal immunization of rUreB reduced $\mathrm{Hp}$ colonization in the stomach, which was closely related to the increased rUreB-specific Th17 cell responses. This suggests that UreB is an important protein able to elicit Th17 cell responses against Hp, both in vivo and in vitro [7].

The Th17 cell responses to Hp infection may involve the following pathways. (1) Infected macrophages directly induce cell differentiation. Coculture of CD4+ T cells with Hp-infected macrophages elevated IL-17 and interferon- $\gamma$ secretion, upregulated ROR $\gamma \mathrm{t}$ expression and increased the number of Th17 cells. The expression of CD40, CD80 and CD86 and the secretion of IL-6, TGF- $\beta_{1}$, IL-23 and CCL20 were significantly increased in Hp-stimulated macrophages. The nuclear factor (NF)- $\kappa$ B pathway participated in the production of IL-6, IL-23 and CCL20 from macrophages in response to $\mathrm{Hp}$, and inhibition of the NF- $\mathrm{KB}$ pathway of macrophages 
resulted in less Th17 cell differentiation, suggesting that Hp induces Th17 cell differentiation via infected macrophages, involving the NF- $\mathrm{BB}$ pathway [8]. (2) Activation of the MyD88 pathway. MyD88 is a universal adapter protein of Toll-like receptor pathways, significant to information transmission and inflammation mediation. In their study, Obonyo et al. [9] showed that mRNA expression levels of IL-17A and IL-22 were markedly upregulated in wildtype mice compared to MyD88-/- mice both at 6 and 25 weeks in response to infection with $\mathrm{Hp}$, indicating that the induction of Th17 cell responses depends on MyD88 signaling. Furthermore, reduction in the expression of Th17 cell-dependent intestinal antimicrobial peptide lipocalin-2 was linked with increased bacterial burden in the absence of MyD88 signaling. Thus, evidence shows that MyD88-dependent signaling is required for the host to induce a Th17 cell response for the control of Hp. (3) Chemotaxis by chemokines. Chemokines like B-cell-activating factor may be able to drive Th17 cell responses both indirectly, by creating a pro-Th17 cell cytokine milieu through the involvement of innate immune cells, and directly, via the differentiation of T cells towards the specific profile [10].

\section{The Immune Mechanism of Th17 Cells in Hp Infection}

CD4+ T cells differentiate towards Th17 cells after Hp infection; meanwhile, Th17 cells mediate the immune response to Hp. Gil et al. [2] report that the number of both Th17 cells and Tregs was significantly higher in the $\mathrm{Hp}+$ gastritis group than in the control group, while the balance of Th17 cells/Tregs was biased towards Tregs. Tregs encourage the immunologic escapement of $\mathrm{Hp}$ because of their inhibition of the immune response. In addition, the number of Th17 cells was correlated negatively with the bacterial density and positively with the inflammatory scores of polymorphonuclear and mononuclear cells in the $\mathrm{Hp}+$ gastritis group, suggesting that Th17 cells are significantly involved in the clearance of Hp. Kao et al. [11] showed that mucosal CD11c+ dendritic cells (DCs) were located near the surface of normal gastric epithelium and that their number increased after Hp infection. This study of the direct interaction of DCs with Hp showed a Treg-skewed response. In vivo Treg skewing by adoptive transfer of Hp-pulsed DCs reduces the ratio of gastric IL-17/Foxp3 mRNA expressions. The depletion of CD25+ Tregs results in early reduction of Hp density, which is correlated with enhanced peripheral Hp-specific Th17 cell, but not Th1 cell, response. Thus, Hp alters the DC-polarized Th17 cell/Treg balance towards a Treg-biased response, which suppresses the effective induction of Hp-specific Th17 cell immunity. Regulatory DCs can down-mediate the immune response and mediate peripheral tolerance, which is closely related to their immature or semimature state. There is a two-way regulation between DCs and Tregs: DCs can induce amplification and inducible Tregs, while Tregs inhibit the combination of DCs and Th cells and suppress the ability of the DCs of activation, maturation and stimulation to T-cell proliferation. Oertli et al. [12] showed that DCs exposed to Hp in vitro or in vivo failed to induce T-cell effector functions. Instead, they efficiently induced expression of the forkhead transcription factor FoxP3, the master regulator of Tregs, a process which requires IL-18.

In conclusion, $\mathrm{Hp}$ infection inducts the Th17 cell/Treg balance bias towards Tregs by suppressing DC maturation or re-encoding. Even though the amount of both Tregs and Th17 cells is increased, it ends with immunologic escapement, since the Tregs are in a dominant position. In such a process, the number of Th17 cells is correlated negatively with bacterial density and positively with inflammation, suggesting that the Th17 cell response participates in the clearance of $\mathrm{Hp}$, which involves releasing IL-17, recruiting and activating neutrophils, and directly removing $\mathrm{Hp}[13,14]$.

Meanwhile, though the immune responses mediated by Th17 cells play an important role in Hp clearance, they somehow also promote the occurrence and development of Hp-related 
diseases, including the following pathways: (1) IL-17 released by Th17 cells activates ERK1/2MAPK and promotes the production of IL-8, which is considered to induce inflammation, damages the gastric mucosa and is closely related to tumorigenesis [15-17]. (2) Activation and expansion of Th1 cell response, which encourages the immunologic escapement of $\mathrm{Hp}$, whereas at the same time, the cytokines, reactive oxygen species and nitric oxide released by this process further expand the inflammation and oxidative stress, thus exacerbating mucosal damage and epithelial cell apoptosis [18-20].

It should be noted that children's immune responses to Hp infection are different from those of adults. Though children are more likely to be infected by Hp than adults, it turns out that they have a low rate of Hp-related diseases like gastritis and peptic ulcer disease. A study of Bangladeshi infants, children and adults found that infant cells produced significantly more IL-17 compared to adult cells after Hp membrane preparation stimulation, and adult cells produced the least. The strong IL-17 production in infants after Hp membrane preparation stimulation was paralleled by a significantly higher production of the IL-17-promoting cytokine IL-1 $\beta$ from infant compared to adult peripheral blood mononuclear cells and monocytes [21].

There is also some research showing that peptic ulcer disease is less frequent in children and less frequently associated with Hp infection. When comparing infected subjects to controls, densities of neutrophils and CD20 cells in the lamina propria increased in all age groups, whereas the CD3 cells increased only in patients aged $>8$ years and CD8 cells only in

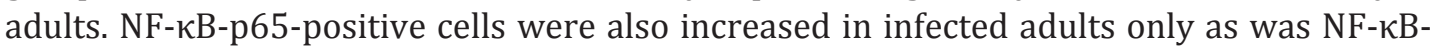
binding activity. A positive correlation was found between age and densities of neutrophils and CD3, but not of CD8 or CD20 [22]. Children's lower level of Th17 cells and higher level of Tregs compared to those of adults may be explained by the immunosuppressive and Hp colonization advantage caused by the Treg dominance of the Th17 cell/Treg balance [2, 11, 23]. Homoplastically, Michalkiewicz et al. [24], observing the varieties of cytokines and the complement in gastric mucosa of Hp-infected children, found out that children's immune response to Hp infection shows specific characters: (1) Th1 expression profile, (2) lack of mRNA overexpression of natural immunity receptors, and (3) strong anti-inflammatory activities in the gastric mucosa, possibly resulting from increased activity of anti-inflammatory M2 macrophages.

Though the reason for the different responses to $\mathrm{Hp}$ infection between children and adults is still not clear, it could be speculated that the Th1- and Treg-dominated immune response of children may show less digestive manifestation but is propitious to Hp escapement and, thus, induces long-term inflammation. It is newly accepted that children's Hp infection not only induces digestive diseases but also other diseases like iron deficiency, diarrhea, growth retardation and idiopathic thrombocytopenic purpura $[25,26]$. Thus, it is worth paying more attention to these mechanisms and to intervene in the case of Hp infection in children.

\section{Diseases Related to Th17 Cells and Hp Infection}

Hp infection is an important pathological factor of gastritis and peptic ulcer disease, while moderate/severe intestinal metaplasia and dysplasia of gastritis are considered as precancerous lesions of gastric cancer. Among the cytokines related to Th17 cells, IL-21 and TGF- $\beta$ are those mostly connected to gastritis and peptic ulcer disease [27]. The level of IL-17 is significantly increased in gastric mucosa of Hp-infected patients [5]. One study on the biopsies of gastritis children showed that the number of Th17 cells was significantly higher in the $\mathrm{Hp}+$ gastritis group than in the control group, and the number of Th17 cells correlated 
negatively with the bacterial density and positively with the inflammatory scores of polymorphonuclear and mononuclear cells in the Hp+ gastritis group [2].

The WHO defines Hp as a class I carcinogen of gastric cancer. It has been observed that the accumulation of Th17 cells and Tregs in the tumor microenvironment gradually increase according to disease progression, leading to an imbalance in Th17 cells/Tregs in gastric cancer patients. TGF- $\beta$ and IL- 6 present in the gastric cancer microenvironment promote the differentiation and expansion of Th17 cells, and increased numbers of Th17 cells promote tumor progression through the promotion of inflammation by secretion of IL-17. After that, IL-7 promotes the secretion of IL-8, which could induce proliferation and migration of cancer cells $[16,28]$. Gastric myofibroblasts/fibroblasts (GMF) are abundant class II major histocompatibility complex-expressing cells that act as novel antigen-presenting cells. Here, we have demonstrated the accumulation of Th17 cells in Hp-infected human tissues and in the gastric tumor microenvironment. GMF isolated from human gastric cancer and $\mathrm{Hp}$-infected tissues cocultured with CD4+ T cells induce substantially higher levels of Th17 cells than GMF from normal tissues in an IL-6-, TGF- $\beta$ - and IL-21-dependent manner. Th17 required interaction with class II major histocompatibility complex on GMF for activation and proliferation. These studies suggest that Th17 cells are induced during both Hp infection and gastric cancer in the inflammatory milieu of gastric stroma and may be an important link between inflammation and carcinogenesis [29]. It should be noted that the Th17 cell immunity still lasts in the serum and mucosa even after the eradication of Hp [5], and the persistence of IL-17A and IL-17F is considered to increase the risk of cancer [30]. In addition, it has been reported that the secreted peptidyl-prolyl-cis, trans-isomerase of Hp (HP0175) is able to drive gastric Th17 cell response, and the mechanisms of carcinogenesis may include the promotion of proinflammatory low cytotoxic tumor-infiltrating lymphocyte response, matrix degradation and proangiogenic pathways [31].

\section{Conclusion}

In conclusion, Hp infection leads to an increased number of Th17 cells. At the same time, Th17 cell response participates in the occurrence and development of Hp-related diseases by eliminating $\mathrm{Hp}$ and other mechanisms. The prognosis of chronic bacterial infection is normally determined by host immune response. Being different from other pathogens, $\mathrm{Hp}$ is able to exist for a long time in the host body by immunologic escapement and to induce sustained inflammation, while the host's immune response cannot eliminate Hp but encourages its colonization.

Though there is substantive research on the mechanisms of the immunologic escapement of Hp, it still needs to be clarified. Numerous studies indicate that Hp infection leads to the immunologic derangement of T cells, and the disorder of Th1/Th2 cells, and that Th17 cells/ Tregs may be related to the host defense and Hp colonization; of all these, Th17 cell immune response seems to be quite important. After Hp infection, CD4+ T cells differentiate towards Th17 cells by directly inducing infected macrophages, activating the MyD88 pathway, and by driving chemokines like B-cell activating factor, while at the same time, Th17 cell responses play an important role in Hp infection. On the one hand, they participate in the clearance of Hp by recruiting neutrophilic granulocytes and other mechanisms, while on the other hand, they expand the inflammation, exacerbate mucosal damage and even induce carcinogenesis; thus, the variation in the balance may influence the prognosis of Hp infection. Clarification of the involvement of Th17 cells is needed to provide a new strategy and more specific targets for the treatment of Hp infection. 


\section{Acknowledgement}

This work was supported by grants from the Program of Three-Year Action Foundation of Shanghai (ZY3-JSFC-2-1039) and the National Basic Science Personal Training Foundation of China (No. J1103607).

\section{References}

1 Kayhan B, Arasli M, Eren H, Aydemir S, Aktas E, Tekin I: Analysis of peripheral blood lymphocyte phenotypes and Th1/Th2 cytokines profile in the systemic immune responses of Helicobacter pylori infected individuals. Microbiol Immunol 2008;52:531-538.

2 Gil JH, Seo JW, Cho MS, Ahn JH, Sung HY: Role of Treg and TH17 cells of the gastric mucosa in children with Helicobacter pylori gastritis. J Pediatr Gastroenterol Nutr 2014;58:245-251.

3 Iwakura Y, Ishigame H: The IL-23/IL-17 axis in inflammation. J Clin Invest 2006;116:1218-1222.

4 Nurgalieva ZZ, Conner ME, Opekun AR, Zheng CQ, Elliott SN, Ernst PB, et al: B-cell and T-cell immune responses to experimental Helicobacter pylori infection in humans. Infect Immun 2005;73:2999-3006.

5 Serelli-Lee V, Ling KL, Ho C, Yeong LH, Lim GK, Ho B, et al: Persistent Helicobacter pylori specific Th17 responses in patients with past H. pylori infection are associated with elevated gastric mucosal IL-1beta. PLoS One 2012; 7:e39199.

6 Lina TT, Pinchuk IV, House J, Yamaoka Y, Graham DY, Beswick EJ, et al: CagA-dependent downregulation of B7-H2 expression on gastric mucosa and inhibition of Th17 responses during Helicobacter pylori infection. J Immunol 2013;191:3838-3846.

7 Zhang JY, Liu T, Guo H, Liu XF, Zhuang Y, Yu S, et al: Induction of a Th17 cell response by Helicobacter pylori urease subunit B. Immunobiology 2011;216:803-810.

8 Zhuang Y, Shi Y, Liu XF, Zhang JY, Liu T, Fan X, et al: Helicobacter pylori-infected macrophages induce Th17 cell differentiation. Immunobiology 2011;216:200-207.

9 Obonyo M, Rickman B, Guiney DG: Effects of myeloid differentiation primary response gene 88 (MyD88) activation on Helicobacter infection in vivo and induction of a Th17 response. Helicobacter 2011;16:398-404.

10 Munari F, Fassan M, Capitani N, Codolo G, Vila-Caballer M, Pizzi M, et al: Cytokine BAFF released by Helicobacter pylori-infected macrophages triggers the Th17 response in human chronic gastritis. J Immunol 2014; 193:5584-5594.

11 Kao JY, Zhang M, Miller MJ, Mills JC, Wang B, Liu M, et al: Helicobacter pylori immune escape is mediated by dendritic cell-induced Treg skewing and Th17 suppression in mice. Gastroenterology 2010;138:1046-1054.

12 Oertli M, Sundquist M, Hitzler I, Engler DB, Arnold IC, Reuter S, et al: DC-derived IL-18 drives Treg differentiation, murine Helicobacter pylori-specific immune tolerance, and asthma protection. J Clin Invest 2012;122: 1082-1096.

$13 \mathrm{Zu} \mathrm{Y,} \mathrm{Cassai} \mathrm{ND,} \mathrm{Sidhu} \mathrm{GS:} \mathrm{Light} \mathrm{microscopic} \mathrm{and} \mathrm{ultrastructural} \mathrm{evidence} \mathrm{of} \mathrm{in} \mathrm{vivo} \mathrm{phagocytosis} \mathrm{of} \mathrm{Helico-}$ bacter pylori by neutrophils. Ultrastruct Pathol 2000;24:319-323.

14 DeLyria ES, Redline RW, Blanchard TG: Vaccination of mice against H. pylori induces a strong Th-17 response and immunity that is neutrophil dependent. Gastroenterology 2009;136:247-256.

15 Siregar GA, Halim S, Sitepu VR: Serum TNF- $\alpha$, IL-8, VEGF levels in Helicobacter pylori infection and their association with degree of gastritis. Acta Med Indones 2015;47:120-126.

16 Lee KE, Khoi PN, Xia Y, Park JS, Joo YE, Kim KK, et al: Helicobacter pylori and interleukin-8 in gastric cancer. World J Gastroenterol 2013;19:8192-8202.

17 Sebkova L, Pellicano A, Monteleone G, Grazioli B, Guarnieri G, Imeneo M, et al: Extracellular signal-regulated protein kinase mediates interleukin 17 (IL-17)-induced IL-8 secretion in Helicobacter pylori-infected human gastric epithelial cells. Infect Immun 2004;72:5019-5026.

18 Shi Y, Liu XF, Zhuang Y, Zhang JY, Liu T, Yin Z, et al: Helicobacter pylori-induced Th17 responses modulate Th1 cell responses, benefit bacterial growth, and contribute to pathology in mice. J Immunol 2010;184:51215129.

19 Robinson K, Kenefeck R, Pidgeon EL, Shakib S, Patel S, Polson RJ, et al: Helicobacter pylori-induced peptic ulcer disease is associated with inadequate regulatory T cell responses. Gut 2008;57:1375-1385.

20 Siomek A, Rytarowska A, Szaflarska-Poplawska A, Gackowski D, Rozalski R, Dziaman T, et al: Helicobacter pylori infection is associated with oxidatively damaged DNA in human leukocytes and decreased level of urinary 8-oxo-7,8-dihydroguanine. Carcinogenesis 2006;27:405-408.

21 Bhuiyan TR, Islam MM, Uddin T, Chowdhury MI, Janzon A, Adamsson J, et al: Th1 and Th17 responses to Helicobacter pylori in Bangladeshi infants, children and adults. PLoS One 2014;9:e93943.

22 Bontems P, Aksoy E, Burette A, Segers V, Deprez C, Mascart F, et al: NF-kappaB activation and severity of gastritis in Helicobacter pylori-infected children and adults. Helicobacter 2014;19:157-167.

23 Serrano C, Wright SW, Bimczok D, Shaffer CL, Cover TL, Venegas A, et al: Downregulated Th17 responses are associated with reduced gastritis in Helicobacter pylori-infected children. Mucosal Immunol 2013;6:950-959. 
24 Michalkiewicz J, Helmin-Basa A, Grzywa R, Czerwionka-Szaflarska M, Szaflarska-Poplawska A, Mierzwa G, et al: Innate immunity components and cytokines in gastric mucosa in children with Helicobacter pylori infection. Mediators Inflamm 2015;2015:176726.

25 Queiroz DM, Rocha AM, Crabtree JE: Unintended consequences of Helicobacter pylori infection in children in developing countries: iron deficiency, diarrhea, and growth retardation. Gut Microbes 2013;4:494-504.

26 Cheng YS, Kuang LP, Zhuang CL, Jiang JD, Shi M: Effects of cytotoxin-associated gene A (CagA) positive Helicobacter pylori infection on anti-platelet glycoprotein antibody producing B cells in patients with primary idiopathic thrombocytopenic purpura (ITP). Pak J Med Sci 2015;31:121-126.

27 Shamsdin SA, Alborzi A, Rasouli M, Hosseini MK, Bagheri Lankrani K, Kalani M: Alterations in Th17 and the respective cytokine levels in Helicobacter pylori-induced stomach diseases. Helicobacter 2015;20:460-475.

28 Li Q, Chen J, Liu Y, Zhao X, Tan B, Ai J, et al: Prevalence of Th17 and Treg cells in gastric cancer patients and its correlation with clinical parameters. Oncol Rep 2013;30:1215-1222.

29 Pinchuk IV, Morris KT, Nofchissey RA, Earley RB, Wu JY, Ma TY, et al: Stromal cells induce Th17 during Helicobacter pylori infection and in the gastric tumor microenvironment. PLoS One 2013;8:e53798.

30 Yu H, Sun S, Liu F, Xu QH: Meta-analysis of associations between interleukin-17 gene polymorphisms and risk of gastric cancer. Asian Pac J Cancer Prev 2014;15:8709-8713.

31 Amedei A, Munari F, Bella CD, Niccolai E, Benagiano M, Bencini L, et al: Helicobacter pylori secreted peptidyl prolyl cis, trans-isomerase drives Th17 inflammation in gastric adenocarcinoma. Intern Emerg Med 2014;9: 303-309. 